\title{
Energy-Efficient Transaction Management for Real- Time Mobile Databases in Ad-hoc Network Environments
}

\author{
Le Gruenwald, Shankar M. Banik \\ University of Oklahoma \\ School of Computer Science \\ Norman, OK 73019 \\ \{ggruenwald, smbanik\}@ou.edu
}

\begin{abstract}
In an ad-hoc mobile network architecture, all the mobile hosts (MHs) are connected with each other through a wireless network that has a frequently changing topology. This type of architecture is used in many applications such as battlefields and disaster recovery where it is difficult or not feasible to depend on a static wired communication infrastructure. These applications are usually time-critical where many of their transactions must not only be executed correctly but also within their deadlines. In addition, the MHs in this environment are not connected to unlimited power supplies and may store data that can be shared by other MHs. Existing mobile database transaction management techniques do not consider the ad-hoc network characteristics, real-time constraints and energy limitation. This paper identifies the issues that need to be addressed in this new environment and proposes approaches for solutions.
\end{abstract}

\section{Introduction}

The Transaction Manager of a Mobile Multi-Database Management System (MMDBMS) is responsible for providing reliable and consistent units of computing to its users, and must address the issues of disconnection and migration of users. This paper provides a transaction management technique that considers energy consumption and real time requirements for an ad-hoc network MMDBMS. In this environment all MHs are roaming and the network that interconnects these MHs is a wireless network with a frequently changing topology.

\section{Proposed Solution Approaches}

MHs in our architecture can be classified into two groups: 1) computers with reduced memory, storage, power and computing capabilities which we will call Small Mobile Hosts (SMHs) and 2) classical workstations equipped with more storage, power, communication and computing facilities than SMHs which we will call Large Mobile 
Hosts (LMHs). Every MH will have a radius of influence. The MHs will operate in three modes to reduce the energy consumption: active, doze and sleep mode.

Transactions are classified into two categories: firm and soft [3]. Firm transactions must be aborted if they miss their deadlines while soft transactions still can be executed after their deadlines have expired.

In our transaction management technique, we have considered time as the most important factor in handling firm transactions and energy in handling soft transactions. So the SMH will submit a firm transaction to the nearest $\mathrm{LMH}$ and a soft transaction to the LMH which has the highest available energy. Now if the LMH is in the active mode, it will receive the transaction and start processing the transaction. If the $\mathrm{LMH}$ is in the doze mode, and the transaction is firm, it will wake up and start processing the transaction. But if the $\mathrm{LMH}$ is in the sleep mode, it will not be able to receive the transaction. In this case the requesting $\mathrm{SMH}$ will wait for some time period. If it does not receive the result of the transaction in this time period, it will again check its local database to find the next nearest LMH (for firm transactions) and submit the transaction.

Each LMH will have three components: Transaction Scheduler (TS), Transaction Coordinator (TC) and Transaction Manager (TM). The LMH after receiving a transaction from an SMH will pass the transaction to TS which will use a real-time energy-efficient dynamic scheduling algorithm to schedule transactions. The scheduling algorithm will organize the transactions in a queue that reflects their priorities of execution. Then the first transaction from the queue will be taken by the TC. The TC will check the required data items for this transaction after consulting the 'Global Schema'. If all the data items required by the transaction are available in this LMH, it will pass the transaction to its TM. If all the data items are not available in this LMH, the TC will find the LMHs which contain the required data items from the 'Global Schema'. Then it will divide the global transaction into sub-transactions and submit the corresponding sub-transactions to the participating LMHs. The participating LMHs after processing the sub-transactions will inform the coordinator LMH. The coordinator LMH will use PGSG algorithm [2] to verify Atomicity/Isolation properties of the global transaction. Then it will submit the result of the transaction to the requesting SMH. The requesting SMH after receiving the result of the transaction will send an acknowledgement to the LMH.

Detailed description of the transaction management technique can be found in [1]

\section{References}

1. Banik, Shankar M., L., Gruenwald.: Power Aware Management of Mobile Real-Time Database Transactions in Ad-hoc Networks. Technical Report, School of Computer Science, University of Oklahoma, October 2000.

2. Dirckze, R., L, Gruenwald : A Pre-serialization Transaction Management Technique for Mobile-Multi-databases. To appear: Special Issue on Software Architecture for Mobile Applications, MONET 2000.

3. Gruenwald, L., et al. : Database Research at The University of Oklahoma. ACM SIGMOD RECORD, Vol. 28, No. 3, September 1999. 